\title{
NOTA SOBRE LA PRESENCIA DE PHYTOLACCA RUGOSA (PHYTOLACCACEAE) EN LA FLORA DEL VALLE DE MÉXICO
}

\author{
Ramiro Cruz Durán \\ JAIME JIMÉNEZ RAMÍREZ \\ Y \\ Ma. Elena García Granados \\ Herbario FCME \\ Departamento de Biología \\ Facultad de Ciencias \\ Universidad Nacional Autónoma de México \\ Apdo. postal 70-399 \\ 04510 México, D. F. \\ cdr@minervaux2.fciencias.unam.mx
}

\section{RESUMEN}

Se registra para la flora del Valle de México a Phytolacca rugosa A. Braun \& C. D. Bouché. La especie se ha encontrado en bosque de Quercus perturbado en Cañadas de Santa Fe, en las cercanías de San Lorenzo Tepeaculco, Delegación Cuajimalpa de Morelos y en Bosques del Pedregal, Delegación de Tlalpan, Distrito Federal. También se registran en esta planta anomalías florales semejantes a las observadas en $P$. icosandra $L$.

Palabras clave: anomalía floral, México, Phytolacca rugosa, Phytolaccaceae, Valle de México.

\section{ABSTRACT}

Phytolacca rugosa A. Braun \& C. D. Bouché is reported for the flora of the Valley of Mexico. This species inhabits in secondary vegetation derived from oak forest at Cañadas de Santa $\mathrm{Fe}$, near San Lorenzo Tepeaculco and at Bosques del Pedregal, Distrito Federal. Floral abnormalities similar to those reported for $P$. icosanda $L$. are also recorded in this species.

Key words: floral abnormality, Mexico, Phytolacca rugosa, Phytolaccaceae, Valley of Mexico.

En exploraciones botánicas realizadas en Cañadas de Santa Fe en la Delegación Cuajimalpa de Morelos, D. F. se encontraron ejemplares de Phytolacca rugosa a los 2460 - 2600 m s.n.m. en vegetación secundaria derivada de bosque de Quercus. En estos lugares convive con $P$. icosandra L. y entre los elementos dominantes destacan: Quercus laeta Liebm., Q. crassipes Kunth, Q. mexicana Humb. et Bonpl., Garrya laurifolia Hartw., 
Prunus serotina Ehrh. ssp. capuli (Cav.) McVaugh, Arbutus xalapensis Kunth, Cupressus lindleyi Klotzsch, Crataegus pubescens (Kunth) Steud., Cornus excelsa Kunth, Senecio roldana DC., S. barba-johannis DC., Cestrum thyrsoideum Kunth, Villadia mexicana (Schltdl.) Jacobsen, Salvia elegans Vahl, Sigesbeckia jorullensis Kunth, Loeselia mexicana (Lamb.) Brand, Fragaria mexicana Schltdl. Los individuos encontrados crecen en suelos areno-fangosos.

En Bosques del Pedregal, en la Delegación de Tlalpan, P. rugosa prospera a $2600 \mathrm{~m}$ s.n.m. y se distribuye de manera abundante en restos de bosque de Quercus-Pinus conviviendo con Quercus mexicana Humb. et Bonpl., Q. rugosa Née, Sigesbeckia jorullensis Kunth, Garrya laurifolia Hartw., Tagetes lunulata Ortega, Brassica sp., Solanum cervantesii Lag., Buddleia cordata Kunth y Pinus sp. Las poblaciones observadas se encontraron preferentemente en suelos arcillosos, así como en sustrato basáltico poroso.

Anteriormente Phytolacca rugosa se conocía para México de los estados de Jalisco, Michoacán, Guerrero, Veracruz, Oaxaca y Chiapas, donde crece en bosque de Pinus, bosque de Quercus y vegetación derivada de los mismos (Martínez-García, 1984). Para la flora fanerogámica el Valle de México Rzedowski y colaboradores (2001) señalan sólo la presencia de $P$. icosandra L. como componente de Phytolaccaceae.

$P$. rugosa se presenta como hierba erguida de 0.5 a $1 \mathrm{~m}$ de altura, glabra, de tallos cilíndricos con tonalidades de color púrpura. Hojas subpecioladas, con el haz verde obscuro, el envés claro, oblongo-lanceoladas, de $6.5-12 \mathrm{~cm}$ de largo por $5.5(-6.5) \mathrm{cm}$ de ancho, coriáceas, ápice caudado, base atenuada, con 8(-9) pares de venas secundarias; pecíolo de 1.5-2.8 cm de largo. Inflorescencias terminales y opositifoliares, de 3-6.2 cm de largo por 1.2-2.2 cm de ancho, con flores alternas; pedúnculo erecto, verdoso de 2-6 cm de largo; brácteas persistentes, rojizas, triangulares, de 4-5 mm de largo; pedicelos de $1 \mathrm{~mm}$ de largo; sépalos persistentes, de 2-3 $\mathrm{mm}$ de largo por $2 \mathrm{~mm}$ de ancho, estambres 8, en una serie, filamentos blancos de $1 \mathrm{~mm}$ de largo, anteras amarillas de ca. $1 \mathrm{~mm}$ de largo; ovario con 8 carpelos unidos en la base, globoso, de $1 \mathrm{~mm}$ de diámetro y estilos de ca. $1 \mathrm{~mm}$. Fruto una baya lobulada, guinda-morada, de 5-6 mm de diámetro, con reminiscencias de los estilos; semillas 1 por lóculo, negras, lenticulares, de $3 \times 2 \times$ $1.5 \mathrm{~mm}$ (Fig. $1 \mathrm{a}, \mathrm{b}$ y c).

La presencia de ocho estambres en una serie, ocho carpelos e inflorescencias de no más de $7 \mathrm{~cm}$ de largo, indica que estas poblaciones concuerdan con lo que Rzedowski y Calderón de Rzedowski (2000a) designan como el primer conjunto o grupo, caracterizado por inflorescencias racemosas de menos de $12 \mathrm{~cm}$ de largo, pedicelos de 3 a $8 \mathrm{~mm}$ de largo, 8 a 12 estambres y 4 a 8 carpelos, con distribución entre los 1000 y 2500 m s.n.m., que comprende plantas que habitan el bosque mesófilo de montaña o encinares y pinares húmedos.

Clave para especies de Phytolacca del Valle de México, modificada de Rzedowski y Calderón de Rzedowski (2000b)

Carpelos libres en la parte apical, estilos manifiestamente separados entre sí, brácteas subuladas a lanceoladas, de 1.5 a $4(-5) \mathrm{mm}$ de largo .................................... . rugosa Carpelos connados en un solo cuerpo, estilos conniventes, brácteas lanceoladas, hasta de $7 \mathrm{~mm}$ de largo ........................................................................................ P. icosandra 
Cruz et al.: Nota sobre Phytolacca rugosa en la flora del Valle de México

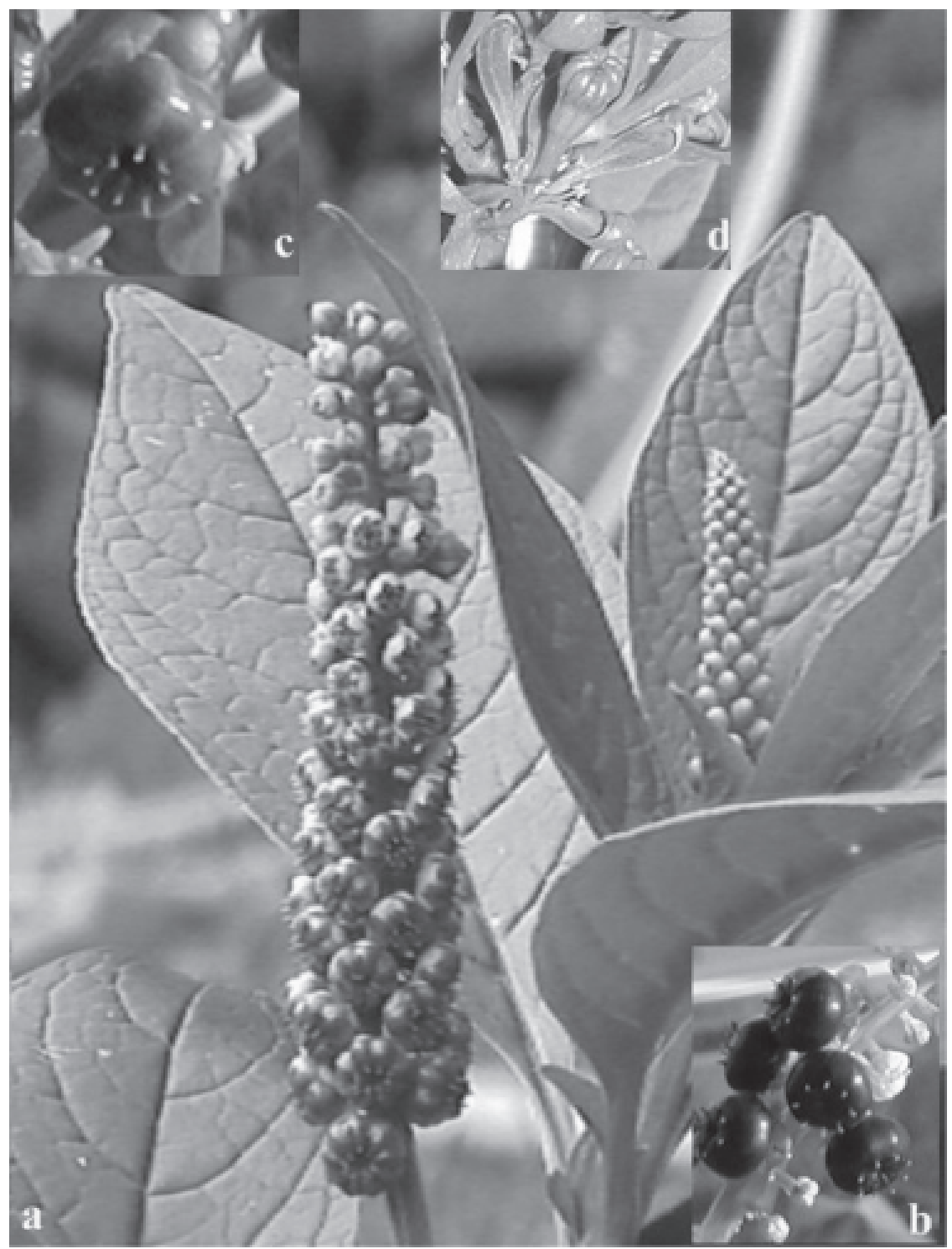

Fig. 1. Phytolacca rugosa A. Braun \& C. D. Bouché. a. Rama con inflorescencia; b. Frutos maduros con reminiscencias de los estilos; c. Pistilos subapocárpicos; d. Anomalía floral: perianto folioso, ovario y base del ovario alargados (a, M. E. García Granados s.n. (FCME); b y c, R. Cruz Durán 5352B (FCME); d, R. Cruz Durán y M. E. García Granados 5511 (FCME)). 
Material examinado: Distrito Federal: Delegación Cuajimalpa de Morelos, cañada Helechos, 0.2 km al N del Panteón Jardín, 19²1'4" 99¹5'31", 2600 m s.n.m., 22.XI.2000, R. Cruz Durán et al. 23CH (FCME); cañada Atzoyapa, $0.2 \mathrm{~km}$ al SE del Panteón Jardín, 2572 m s.n.m., 19²0'52.1" 99¹5'30.3", 22.XI.2000, R. Cruz Durán et al. 133ATX (FCME); cañada Atzoyapa, 0.39 km al SE del Panteón Jardín, 19²0'50.4" 99¹5'20.2", 2578 m s.n.m., 22.XI.2000, R. Cruz Durán et al. 194ATX2 (FCME); Delegación Tlalpan, Col. Bosques del Pedregal, 19¹6'13.5" 99¹4'39.7", 2681 m s.n.m., 27.VIII.2001, R. Cruz Durán y L. Cruz García 5352 (FCME); 23.IX.2001, M. E. García Granados s.n. (FCME); Col. Bosques del Pedregal, 19¹6'13.5" 99¹4'39.7", 2680 m s.n.m., 11.IV.2002, R. Cruz Durán y M. E. García Granados 5511 (FCME).

Un aspecto interesante es la presencia de algunos individuos de $P$. rugosa con anomalías florales similares a las registradas para $P$. icosandra L. por Cruz y Alcántara (2000) (Fig. 1d; documentada por el ejemplar R. Cruz Durán y M. E. García Granados 5511). También en esta especie, en el mismo individuo las mencionadas irregularidades se presentan sólo en algunas inflorescencias, mientras que en otras se producen flores, frutos y semillas normales. Así, se confirman las sospechas de Rzedowski y Calderón de Rzedowski (2000a), quienes consideran al perianto de $P$. heterotepala $\mathrm{H}$. Walt. como posible resultado de anomalía floral de plantas de $P$. rugosa y $P$. icosandra.

Las anomalías florales de las dos especies se han observado en aquellos individuos que están en contacto con edificaciones, donde se ha utilizado una mezcla de cemento y cal como material básico de construcción.

Finalmente para despejar algunas dudas sobre la identidad de ambas especies, se realizó el siguiente ensayo. Un ejemplar de $P$. rugosa ( $R$. Cruz Durán 5352B), colectado a 2680 m s.n.m. en la colonia Bosques del Pedregal fue cultivado en la Ciudad Universitaria (a 2240 m s.n.m.). En forma simultánea un ejemplar de $P$. icosandra de la Ciudad Universitaria ( $R$. Cruz Durán 5352C) se trasladó a Bosques del Pedregal. Notablemente ambas especies conservaron su identidad (Fig. 1b y c).

Material herborizado después de su cultivo. Distrito Federal: Delegación Coyoacán, Facultad de Ciencias, Ciudad Universitaria, 19¹9'26" 99¹0'42", 2240 m s.n.m., 10.X.2002, R. Cruz Durán 5352C (FCME); Delegación de Tlalpan, col. Bosques del Pedregal, 19¹6'13.5" 99¹4'39.7" 2680 m s.n.m., 10.VII.2002, R. Cruz Durán 5352B (FCME).

\section{AGRADECIMIENTOS}

A la M. en C. Martha Martínez Gordillo la revisión crítica del manuscrito. Dos revisores anónimos hicieron importantes recomendaciones.

\section{LITERATURA CITADA}

Cruz, R. y O. Alcántara. 2000. Anormalidad floral en Phytolacca icosandra L. (Phytolaccaceae) en el Pedregal de San Ángel, México, D. F. Acta Bot. Mex. 53: 27-33. 
Martínez-García, J. 1984. Phytolaccaceae. Flora de Veracruz. Fascículo 36. Instituto Nacional de Investigaciones sobre Recursos Bióticos. Xalapa, Ver. México. 41 pp.

Rzedowski, G. C. de, J. Rzedowski y colaboradores. 2001. Flora fanerogámica del Valle de México. 2a. ed. Instituto de Ecología, A. C. y Comisión Nacional para el Conocimiento y Uso de la Biodiversidad. Pátzcuaro, Michoacán. 1406 pp.

Rzedowski, J. y G. Calderón de Rzedowski. 2000a. Notas sobre el género Phytolacca en México. Acta Bot. Mex. 53: 49-66.

Rzedowski, J. y G. Calderón de Rzedowski. 2000b. Familia Phytolaccaceae. Flora del Bajío y de Regiones Adyacentes. Fascículo 91. Instituto de Ecología, A. C. Pátzcuaro, Mich. 32 pp. 